\title{
Biliary cast syndrome in portal hypertensive biliopathy: direct cholangioscopic findings and endoscopic therapy with metal stent
}

Authors

Institutions
Marco Aurelio D’Assuncao ${ }^{1,2}$, Jacobo Velázquez-Aviña' ${ }^{1}$, Leona Council ${ }^{3}$, Klaus Mönkemüller ${ }^{1}$

Basil I. Hirschowitz Endoscopic Center of Excellence, Division of Gastroenterology and Hepatology, University of Alabama at Birmingham, Alabama, United States

${ }^{2}$ Gastrointestinal Endoscopy Unit, Hospital Sirio Libanes, Sao Paulo, Brazil

3 Department of Pathology, University of Alabama at Birmingham, Alabama, United States submitted 5. January 2015 accepted after revision 12. January 2015

\section{Bibliography}

Dol http://dx.doi.org/

10.1055/s-0034-1391670

Published online: 14.4 .2015

Endosc Int Open 2015; 03: E223-E225

(c) Georg Thieme Verlag KG Stuttgart · New York

E-ISSN 2196-9736

\section{Corresponding author}

Klaus Mönkemüller, MD, PhD,

FASGE

Division of Gastroenterology and Hepatology

Basil I. Hirschowitz Endoscopic

Center of Excellence

Endoscopy Unit, JT 664

619 19th

Street $S$

Birmingham, AL 35249

United States

Fax: +12059341578

klaus1@uab.edu

\section{Abbreviations \\ $\nabla$ \\ CT computed tomography \\ $\mathrm{HCV}$ Hepatitis $\mathrm{C}$ virus \\ EHPVO extrahepatic portal venus obstruction \\ ERCP endoscopic retrograde cholangiopan- creatography \\ HCC hepatocellular carcinoma.}

A 61-year-old man with asthma, diabetes mellitus, cirrhosis secondary to chronic hepatitis C virus (HCV) infection, and hepatocellular carcinoma (HCC), was admitted to our hospital because of progressive abdominal pain and jaundice of 7 days duration. On clinical examination, the patient had abdominal distention, tenderness in the right upper quadrant, and jaundice. His hemoglobin was $8.7 \mathrm{mg} / \mathrm{dL}$; hematocrit, $25 \%$; white blood cells, $1.7 \times 10^{9} / \mathrm{L}$; international normalized ratio (INR), 1.4; total bilirubin, $15 \mathrm{mg} / \mathrm{dL}$; direct bilirubin, $7.6 \mathrm{mg} / \mathrm{dL}$; indirect bilirubin, $7.4 \mathrm{mg} / \mathrm{dL}$; and alkaline phosphatase, $65 \mathrm{IU} / \mathrm{L}$. The remaining laboratory parameters were within reference ranges.

A computed tomography scan (CT) revealed cirrhosis, HCC, portal vein thrombosis and choledocholithiasis with intrahepatic filling defects and ductal dilation ( $\bullet$ Fig.1). During endoscopic retrograde cholangiopancreatography (ERCP) marked portal hypertensive gastropathy and duodenopathy was evident. ERCP showed irregular filling defects within the entire common bile duct ( Fig.2). During standard cannulation the wire would not advance into the bile duct because of the presence of the large impacted filling defect. Therefore, a biliary precut-fistulotomy was performed with subsequent extraction of a worm-shaped structure ("biliary cast”) ( $\bullet$ Fig.3). Upon removal of the biliary cast, hemobilia was observed, which was successfully treated by inserting a fully covered self-expanding metal stent (fcSEMS; Wallstent $10 \mathrm{~mm}$ diameter $\times 80 \mathrm{~mm}$ length, Boston Scientific, Massachusetts, United States) ( Fig.4). The length of the stent was chosen based on the diffuse characteristics of the portal biliopathy.

Two-days later, a direct cholangioscopy (DC) using Spyglass (Boston Scientific, Massachusetts, United States) for biliary cleansing was performed. During the DC, the inner mucosal lining of the bile duct was observed, demonstrating a typical snake-skin mucosal pattern consistent with portal hypertensive biliopathy ( Fig.5, - Video 1). The histology showed a biliary cast $(\bullet$ Fig.3c). The clinical condition stabilized and the patient was discharged home one-week later. The stent was removed uneventfully four-weeks later. At the four-month follow-up, he did not have any further bleeding.

\section{Discussion \\ $\nabla$}

Extrahepatic portal venus obstruction (EHPVO) is a common cause of portal hypertension and occurs in up to $40 \%$ of all patients with portal hypertension, but it is not confined to those patients [1]. Similarly, EHPVO can occur in liver cirrhosis. A rare manifestation of portal hypertension is hemobilia, which is believed to be caused by rupture of abnormally dilated veins in and around the bile duct wall that results from elevated portal venous pressure [1]. In our patient, the bleeding likely originated from the diffuse mucosal changes of biliopathy leading to a large blood clot, which mixed with bile, cells, and debris, and was trans-

\section{Video}

The video demonstrates the typical snake-skin pattern of portal hypertensive biliopathy.

online content including video sequences viewable at: www.thieme-connect.de 

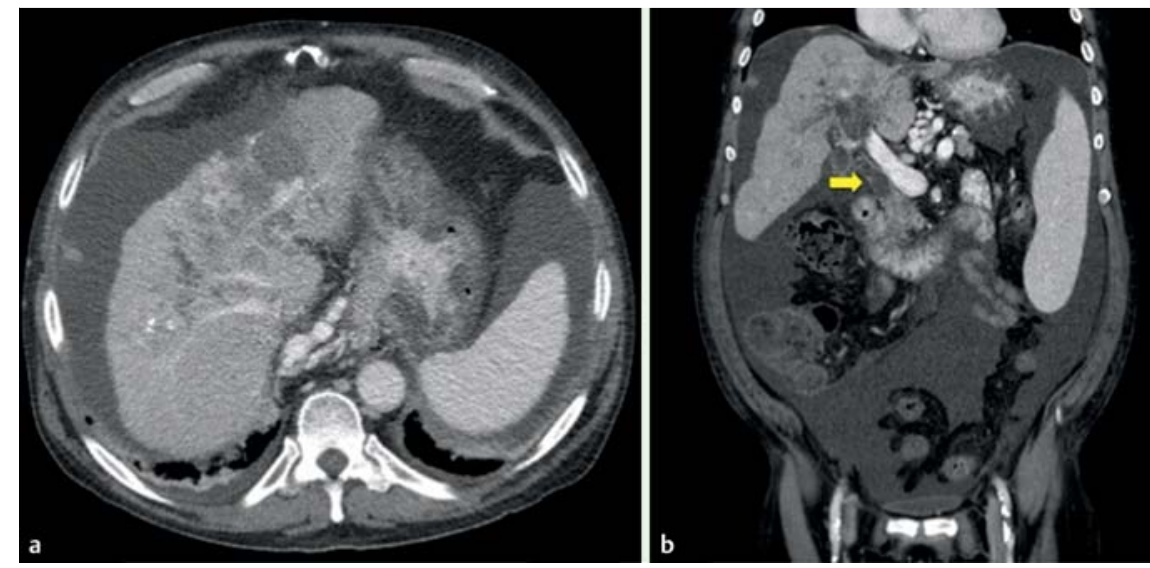

Fig. 1 CT scan. a The CT scan revealed cirrhosis, HCC, portal vein thrombosis and choledocholithiasis with intrahepatic filling defects and ductal dilatation. b Sagital CT showing the distal bile duct filled with a biliary cast.

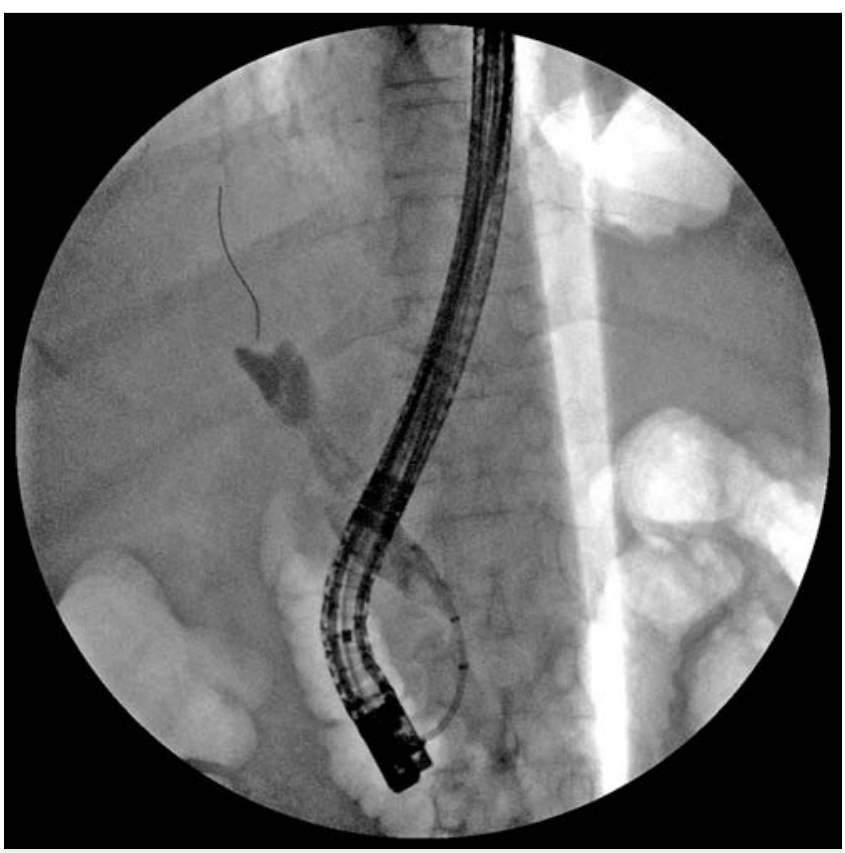

Fig.2 Cholangiography showed irregular filling defects within the entire common bile duct. formed into a biliary cast. Because our patient also had HCC, it is possible that tumor invasion into the bile duct led to hemorrhage. However, the rapid response to tamponade with fcSEMS supports the portal biliopathy as the etiologic factor of the hemobilia. Furthermore, hemobilia due to tumor invasion or variceal rupture usually results in a decrease in hemoglobin, which did not occur in our patient. We suspect that the bleeding was slow but steady because it occurred in portal hypertensive gastropathy. The retained blood clots acted as a lithogenic stimulus, leading to the biliary cast [2,3]. Biliary cast syndrome is an unusual condition that has been reported in liver transplantation, post-cholecystectomy, antiphospholipid antibody syndrome, gallbladder hypocontractility, parenteral nutrition, and biliary infection [2-5]. Diagnosis of biliary cast syndrome is established primarily by ERCP or percutaneous transhepatic cholangiography. The endoscopic therapeutic options include sphincterotomy with balloon or basket extraction, and mechanical and electrohydraulic lithotripsy [2-5]. Other modalities include endoscopic nasobiliary drainage, percutaneous transhepatic drainage, and surgery [25].

In summary, this case report adds to the literature for three important reasons. First, we provided evidence of the existence of mucosal biliopathy by showing the DC view of this disorder. Second, we showed that this condition can lead to formation of a biliary cast and, finally, the concept of treating bleeding biliopathy with fcSEMS was demonstrated.
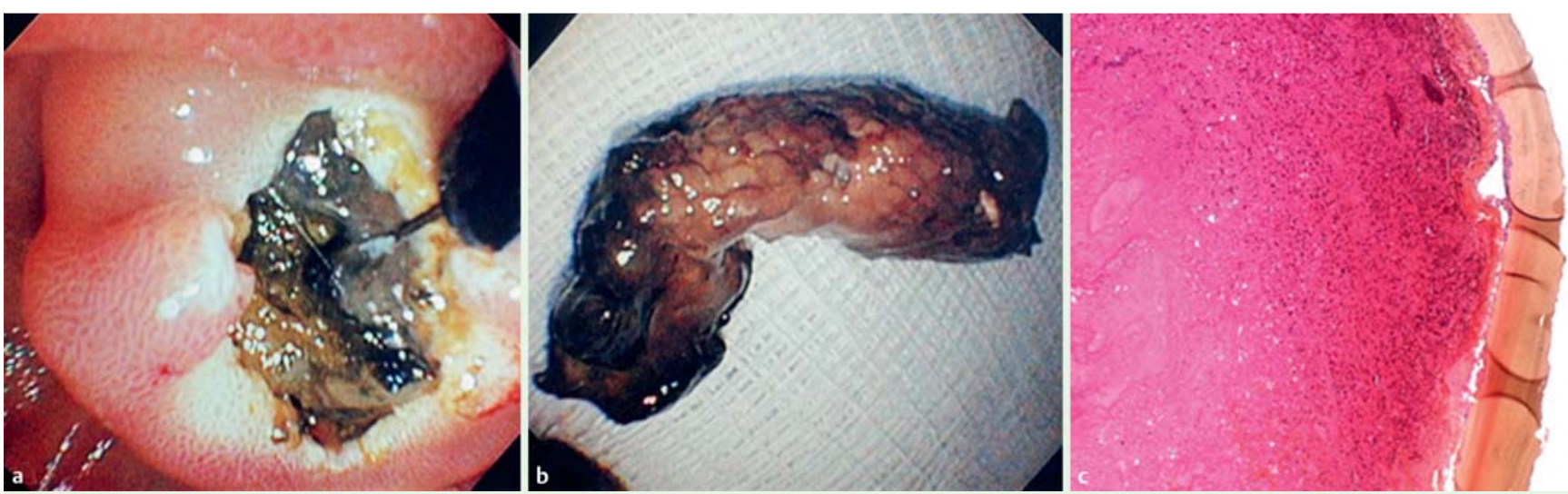

Fig. 3 a Endoscopic extraction of the biliary cast. b Image of the extracted worm-shaped biliary cast. c Histology showed a coagulum with a biliary cast. 

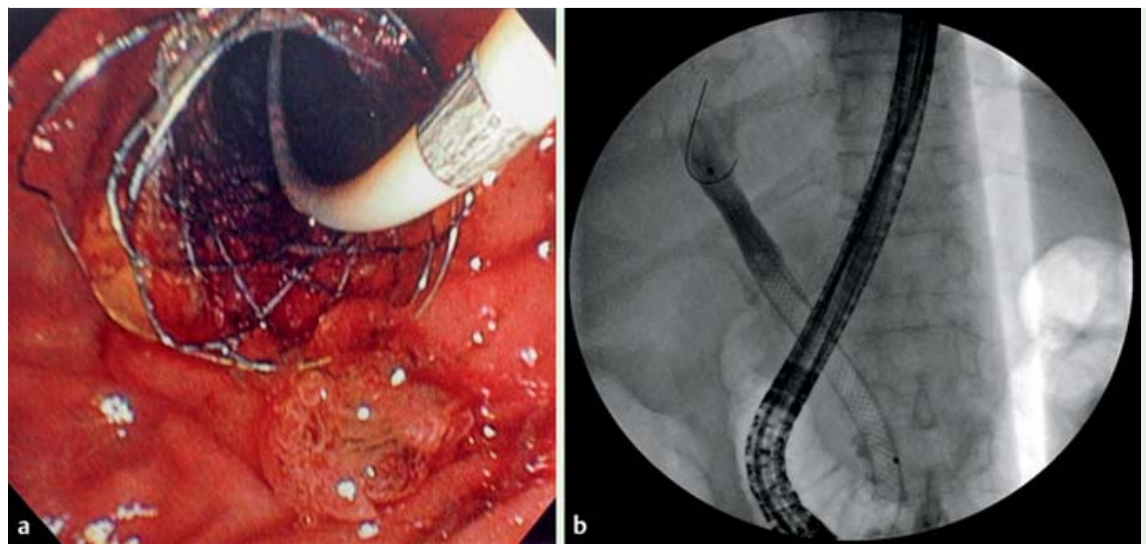

Fig.4 Upon removal of the cast hemobilia was observed, which was successfully treated by inserting a fully covered self-expanding metal stent.

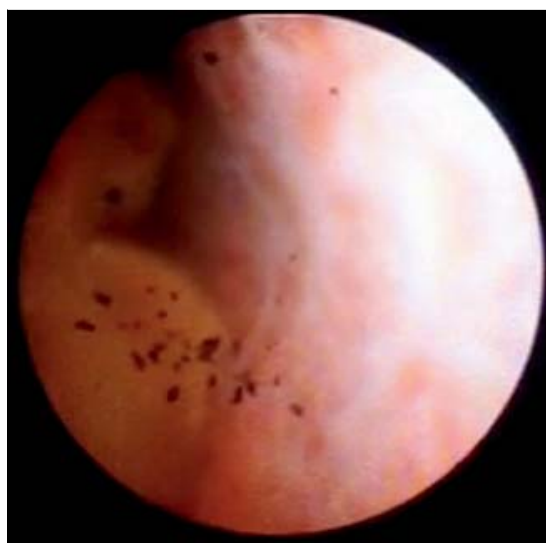

Fig. 5 Endoscopic appearance of portal hypertensive biliopathy.

\section{Acknowledgments}

Marco Aurelio D'Assuncao, MD is a visiting professor at the Basil I. Hirschowitz Endoscopic Center of Excellence. Jacobo VelazquezAviña, MD is the recipient of the Boston Scientific Visiting Gastroenterology Scholar/Fellow Award. This work was performed in part during their visitations at the Basil I. Hirschowitz Endoscopic Center of Excellence, University of Alabama, Birmingham, Alabama, United States.

\section{References}

1 Dhiman RK, Behera A, Chawla YK et al. Portal hypertensive biliopathy. Gut 2007; 56: 1001 - 1008

2 Gleeson FC, Czaja AJ, Baron TH. Successful endoscopic management of biliary cast syndrome in nonliver transplant patients. J Clin Gastroenterol 2008; 42: $752-755$

3 Katsinelos P, Kountouras J. Chatzimavroudis G et al. Combined endoscopic and ursodeoxycholic acid treatment of biliary cast syndrome in a non-transplant patient. World J Gastroenterol 2008; 14: 5223 - 5225

4 Gor NV, Levy RM, Ahn J et al. Biliary cast syndrome following liver transplantation: predictive factors and clinical outcomes. Liver Transpl 2008; $14: 1466$ - 1472

5 Srinivasaiah N, Reddy MS, Balupuri S et al. Biliary cast syndrome: literature review and a single centre experience in liver transplant recipients. Hepatobiliary Pancreat Dis Int 2008; 7: 300-303 